\title{
Preliminary Study on the Implementation of Spiritual Needs Accommodation in Retirement Community Landscape in the Context of Smart Pension
}

\author{
Yanhong Liu ${ }^{1}$ Ting wen ${ }^{2, *}$, Yongdong Liu ${ }^{3}$, Mimi Tsai ${ }^{4}$ and Juan Chen ${ }^{1, *}$ \\ ${ }^{1}$ Nanjing Institute of Technology; \\ ${ }^{2}$ Nanjing University of Finance and Economics; \\ ${ }^{3}$ Institute of Botany, Jiangsu and China Academy of Science; \\ ${ }^{4}$ Queensland University of Technology; \\ *Corresponding author. Email: wen5ting@163.com;894104209@qq.com.
}

\begin{abstract}
Smart Pension aims to cope with severe problems caused by population aging with the help of information technology, which helps the elderly improve their quality of life and demonstrate their value and dignity. Technology and needs accommodation are the cores of Smart Pension, in which the supply of spiritual demand is of great significance to the seniors. This paper focuses on the implementation of spiritual needs accommodation in retirement community landscape in the context of Smart Pension in China. Based on Customer Experience Theory, semi-open interviews was conducted involving two topics of "scenery" and "facilities" from cognition, emotion and behavior, after analyzing, the spiritual needs of elderly Chinese for landscape mainly includes "emotional care" and "cultural care," which are best carried by "intelligent facilities and landscape" and "festivals and cultural landscape," respectively. Based on the founding and the principles of landscape design, we propose several approaches of landscaping for the accommodation of spiritual needs in retirement community with the aid of information technology, which may provide ideas for the construction of comprehensive cares for the elderly.
\end{abstract}

Keywords: Smart pension, The elderly, Spiritual needs, Retirement village, Landscape.

\section{INTRODUCTION}

In 2015, with the increasing growth of aging population, the State Council of the PRC proposed "Advancing the Development of Smart and Healthy Pension Industry" and issued Guidelines on Actively Promoting the "Internet+." In 2017, China further issued Plan for the Advancing the Development of Smart and Healthy Pension Industry, and planned to build 500 normal communities of Smart and Healthy Pension in 5 years. This implies that Smart Pension is of national priority. As a new pension system, Smart Pension is an innovation made to the old pension services and has been put into practice nationwide (Xia, 2020).

"Smart Pension," also known as "intelligent home pension," originated from the Life Trust Foundation of the UK. It takes the advantage of advanced Information Technology and develops Internet of Things platform for the elderly home-based, communities and organization, providing timely, convenient, efficient and intellectualized services, so that elderly users could live a high quality life in their old age at home (Zuo, 2014). The application of intelligent technology, on the one hand, can solve certain medical and nursing problems intensively and effectively, but on the other hand, it also causes pressure on the elderly, resulting in "technophobia". "Technophobia" refers to the fear and rejection of using new technology products or services (Wang, 2014), which is a common phenomenon among elderly users (He, 2020).

Because of the above phenomenon, many studies turn to the connotation of Smart Pension. Zuo Meiyun (2014) clarified that "smart" pension, in terms of semantics, is not just "intelligent" pension, that it should bring more attention to "human being". He stressed that with the help of information technology, elderly users should be able to reflect their own value and dignity while improving their quality of life. In addition, he 
proposed that Smart Pension service should include two aspects: material life and spiritual life. It not only provides sufficient support for the life of the elderly users at the material level, but also enriches the spiritual life of the elderly users, so that the elderly can live a more meaningful life. He Canqun (2020) proposed that "needs" and "technology" are the two core aspects of Smart Pension, and the "needs" of elderly users are the foundation of Smart Pension services, which includes "health needs" and "spiritual and cultural needs". Bai Mei et al. (2016) believe that smart pension should achieve the dual goals consisting of both material and mental health of elderly users. Zhang Quan et al. (2019) suggest four types of core needs for Smart Pension: health, safety, assistance and nursing, among which health needs includes mental health. Although the research shows that the spiritual needs as a part of Smart Pension is of vital importance, the current pension system and pension service institutions pay little attention to the spiritual and cultural needs of the elderly, resulting in the "spiritual poverty" of some old people after retirement (Bai, 2016).

The retirement community this paper mentions is the generalization of real estate for retirement in China (Zhang, 2019; Zhu, 2018). As one of the main environmental carriers for the smart pension, the landscaping in the retirement community so far still emphasizes barrier-free design, focusing on the physical and behavioral safety of elderly users, and lacks research on the supply of spiritual needs. Meeting the full needs of the elderly in landscape is an important part of the retirement community under the humanistic care, but landscaping for the spiritual needs of the seniors has not been fully studied ( $\mathrm{Bu}, 2019)$. The purpose of this paper is to explore the spiritual needs of elderly users with Smart Pension, and discuss how to accommodate spiritual needs for the elderly through landscaping with appropriate technological means.

\section{RESEARCH METHODS}

From the perspective of Customer Experience Theory, Hollebeek, L.D. et al. $(2011,2014)$ proposed that "cognition," "emotion" and "behavior" are three dimensions for analyzing user product experience. We solicited 60 elderly users over 60 years old in Jiangsu, Zhejiang and Shanghai, conducted an semi-open interview from "cognition", "emotion" and "behavior" on the topics of "Scenery" and "Facilities" of landscape in retirement community . "Scenery" relates to aesthetic function and "Facilities" related to practical function. Words, sentences and general ideas related to the spiritual needs of the elderly from the interview content were extracted for cluster analysis. Finally, the types of interpretive spiritual needs and their typical expression model are established. Based on the model and the principles of landscape design, we propose several approaches of landscaping for accommodation of spiritual needs in retirement community with the aid of information technology, which may provide ideas for the construction of comprehensive cares for the elderly.

There were 27 males and 33 females among the 60 interviewees, of whom 24 were 60-69 years old, 18 were 70-79 years old, and 18 were over 80 years old. They were solicited from Nanjing, Shanghai or Hangzhou. A semi-open interview was conducted on the two topics of "scenery" and "facilities" of the landscape of the elderly community.

When asked about the needs in landscape from the perspective of aesthetic function, the vegetation volume, color and style are the three main concerns. "Be situated at the foot of a hill and beside a stream," "abundant plants," "four-season scenery" and "colorful" were generally mentioned by interviewees. More prominent is that about $90 \%$ of the interviewees mentioned that they like colorful plant scenery, such as "colorful spring," "golden autumn." This is consistent with the research results of Chen Yugang et al.(2019) who pointed out that "The color of plants has attracted the attention of the elderly to a certain extent, and the elderly reported that they are more inclined to bright (referring to higher color saturation) colors during the survey."

When asked about the experience related to facilities and space from the perspective of practical functions, the description is complex, involving the needs of individuals, relatives and friends, and communities. On the personal side, the respondents mentioned that "Sitting on a bench is cool, hard, uncomfortable," "Although I prefer to rest or take a walk in a quiet place alone, I dare not because of heart problem." As for relatives and friends, the interviewees mentioned that "Children are always busy with their studies or playing electronic devices, and there are no facilities to attract them." They hope to carry out "meaningful parent-child activities," "joint participation activities" and "facilities suitable for both children and young people." Regarding the community, interviewees mentioned "the favorite place where I do most activities is the lakeside," "I often communicate with friends when we are taking a walk," and also mentioned some key information such as "a variety of activity areas," "cultural atmosphere," "theme activities," "traditional festival celebration"and "hobby development" (Table 1).

Table 1. Summary of interview content

\begin{tabular}{|c|c|}
\hline Aesthetic Function & Practical Function \\
\hline $\begin{array}{c}\text { The vegetation volume } \\
\text { (abundant plants) }\end{array}$ & $\begin{array}{c}\text { Individual (recreational } \\
\text { facilities) }\end{array}$ \\
\hline $\begin{array}{c}\text { Color quality } \\
\text { (bright colors) }\end{array}$ & $\begin{array}{c}\text { Relatives and friends (all-age } \\
\text { facilities) }\end{array}$ \\
\hline
\end{tabular}


Style (mountain and river, $\quad$ Community (cultural and countryside) recreational activities)

\section{INTERVIEW RESULTS AND ANALYSIS}

\subsection{Landscape Aesthetic of the Elderly Reflects their Spiritual and Cultural Trends}

The interviewees' preference for landscape style and artistic conception showed a clear tendency of natural landscape and countryside activities. From an emotional point of view, respondents prefer "natural Chinese garden style" and "natural lake or stream." "Traditional festival activities" have been mentioned many times. The main reason is that "Garden" and "Festival" are more closely related to the living scenes and good hope. Respondents suggested increasing the application of traditional plants which they think represents the human's noble character and making some scenic spots around traditional culture in retirement community. The suggestion of "ecological farmland" was put forward, and it was considered that "planting and picking in garden" had a positive effect on their own health and the growth of their offspring.

Obviously, Chinese natural landscape style and traditional festival landscape are closely related to the cultural belonging and value embodiment of elderly residents. Chinese traditional landscape can be regarded as the container of spiritual culture, while traditional festival activities are the content of spiritual culture. This may be based on traditional cultural cognition and living environment preference of Chinese. Therefore, it is suggested that traditional Chines landscape should be the mainstream of the landscape style in retirement community, focusing on the shaping of cultural landscape and the creation of traditional festival atmosphere.

\subsection{Facilities and Activities Constitute the Medium of Emotional Support for Elderly Residents}

The interview results also show that some interviewees believe that "Building more intelligent facilities and activities for children will attract grandchildren to visit," and "Creating the specific activity spaces for children will enhance their communication with offspring." It can be sure that from the perspective of emotion and cognition, the elderly are eager to contact children and enhance communication with future generations, and recognize that facilities and activities for children are an important means of intergenerational construction.

When it comes to the establishment of a new circle of friends, many people refer to "lakeside" and "sidewalk," which indicates that elderly residents have more opportunities to communicate in natural waterscape areas and in moving state. Therefore, the sites around waterscape and along the footpath should be favorable spaces to promote the emotional construction for elderly residents. Meanwhile, many people suggested that "traditional festival activities" should be carried out in the community, showing their tendency and consistency of the cultural value.

In a word, the support of relatives and friends is an important aspect of the spiritual needs of the elderly residents in retirement community. We can consider setting up an ecological garden, interesting orchard, children's amusement facilities and all-age sport facilities, and pay attention to the uniqueness and interest of the scenery, so as to enhance the fun of visiting relatives and the construction of intergenerational friendship. It should be encouraged to actively carry out the construction of traditional festivals and cultural landscape, and promote the coordinated development of elderly user value and community culture.

\subsection{Types and Representative Expression Carriers of Spiritual Needs in Landscape}

Based on the above analysis, the topics about aesthetic and practical function of the landscape in the retirement community lead to the need of emotion care which includes the parent-child relationship and a new circle of friends, as well as the need of cultural care embodied in the tendency of traditional culture and value. At the same time, cluster analysis carried out on the descriptive content of interviews put forward the representative expressions of spiritual needs in landscape: intelligent facilities and landscape, festival and cultural landscape (Figure 1). 


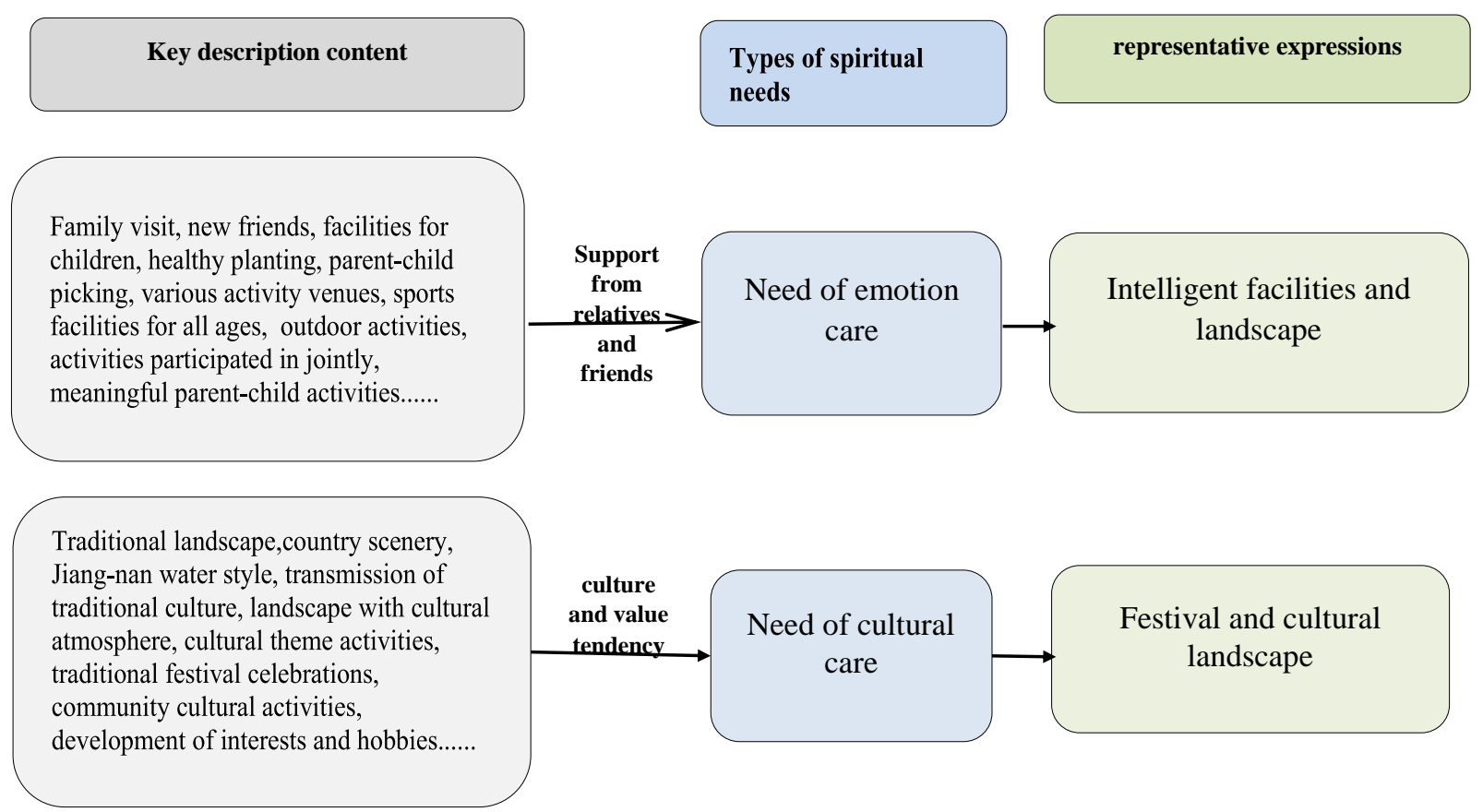

Figure 1 Types and representative expressions of spiritual needs in retirement community landscape. Source: This study.

\section{LANDSCAPE DESIGN APPROACHES}

\subsection{Emotional Support Design Approaches based on Intelligent Facilities}

Outdoor facilities are the basic functional elements of landscape in retirement community. With the growth of age, the physical condition of the elderly generally declines. Most outdoor facilities are stereotyped integrated design (He, 2018). Although the intelligent indoor facilities in retirement community have been fully developed, the special outdoor facilities for the elderly have not attracted enough attention. Therefore, intelligent facilities need be enhanced.

Elderly residents have a limited range of activity and are highly dependent on rest facilities. It is suggested that the intelligent seats should be used to adjust the temperature, softness, back angle and sunshine orientation in retirement community. According to the elderly residents with different health conditions, it is suggested to design outdoor fitness facilities at different degrees, and equipped with auxiliary sports and health detection system to, set the target value and encourage the elderly to achieve goals and experience the sense of achievement, under the premise of safety. It is recommended to install monitoring and guidance devices on sidewalks and relatively independent spaces where elderly residents like to stay. (It monitors risks, records the physical indexes of individual elderly, utilizes cloud data to analyze their habit and preference, and makes customized workout plan and provides instructions In these places where the elderly often go, simulating the natural sound,light and environmental effects can also be used to stimulate the sensory experience of the elderly by Virtual Reality.

The landscape design in a retirement community should not only emphasize the customization for senior people, but also fully consider the needs of users outside the elderly group, and pay more attention to the design of parent-child space, parent-child activities and facilities. At present, in children's activity spaces, high intelligent and high participation parent-child amusement facilities have been effectively developed, and sport facilities supported by cloud technology have also entered people's vision. These children's intelligent facilities and all-age fitness facilities should also become important landscape factors in a retirement community, which can attract the elderly and the younger generation to realize inter-generational mutual assistance and help the elderly overcome their fear of technology. 
Table 2. Emotional support design approaches based on intelligent facilities

\begin{tabular}{|c|c|}
\hline Design approaches & Aims \\
\hline Provide emotional intelligent outdoor recreation facilities. & Gain a sense of physical and mental security. \\
\hline $\begin{array}{c}\text { Provide intelligent outdoor sports facilities for the elderly } \\
\text { Utilize VR to add interesting elements to the spaces, such } \\
\text { as birdsong, streams, fog, etc. }\end{array}$ & $\begin{array}{c}\text { Stimulate the interest and curiosity of the elderly. } \\
\text { sense of achievement. }\end{array}$ \\
\hline Provide children's intelligent entertainment facilities. & Attract children and bring vitality to the elderly. \\
\hline $\begin{array}{c}\text { Provide all age sports facilities supported by cloud } \\
\text { technology. }\end{array}$ & $\begin{array}{c}\text { Attract relatives and friends and enhance the relationship } \\
\text { between generations. }\end{array}$ \\
\hline Provide intelligent facilities based on HCIT & $\begin{array}{c}\text { Attract them to participate in outdoor exercise and } \\
\text { communicate actively }\end{array}$ \\
\hline
\end{tabular}

According to the interview results, elderly users have a significant demand for the establishment of a new circle of friends. Zuo Meiyun (2020) also confirmed in the demand model of smart pension based on data analysis that the elderly pay more attention to social interaction and entertainment in their life after retirement. In addition, the analysis in needs of different types of elderly for smart elderly services shows that the social need is the highest among all types of elderly who have used intelligent services. The possible reason is that most of the elderly use smart phones or computers for social activities such as online chat. From this conclusion, we can see that the elderly residents have strong social desire, and they rely on intelligent media to realize this desire to some extent. It is well known that online social has a negative impact on the health of the elderly. It is suggested that the Human-Computer Interaction Techniques (HCIT) be applied to landscaping, and interactive design of intelligent facilities that fit the elderly be concentrated on to attract them to participate in outdoor exercise and communicate more actively (Table 2 ).

\subsection{Design Approaches for Spiritual and Cultural Value based on the Creation of Artistic Conception in Landscape}

According to the interview results, Chinese idealliving environment--countryside style (Tian-yuan style), is still the pursuit of modern pension environment. Traditionalt natural Chinese landscape is still one of the landscape archetype welcomed by the elderly. Chinese traditional culture has a deep-rooted influence on the ideas and aesthetic standards of the elderly, especially for the traditional festivals and classical gardens, and the elderly wish to develop and inherit the traditional culture spontaneously as well.
Therefore, it is suggested to respect the traditional culture and highlight the status of traditional Chinese landscape in retirement community.

Due to physiological factors that the elderly are less sensitive to things, bright colors are more likely to stimulate the elderly, and bright colors can make them feel warm and energetic(Jia, 2012). In the configuration of landscape plant, Hemerocallis fulva, Pyracantha fortuneana and other colorful plants can be frequently used (Chen, 2019). It is suggested that flower shrubs should be arranged to create a flower-sea scene, which will create an atmosphere for the traditional activities such as "searching for plum blossom in the snow (ta xue xun mei)" and "picking lotus on a canoe (fan zhou cai lian)." Armed with Big data platform and landscape genealogy, we can predict the flowering phase and regulate the proportion of flower color, and exhibit the charisma and culture of the plants.

"Modeling Heaven and Earth" (fa tian xiang di) is the design philosophy of traditional Chinese garden, which is in line with the Chinese agreement to the theory that "Man is an integral part of nature (tian ren he yi)." With the help of natural scenery to express the inner feelings, sentiments and values, and thus forming the expression method of Chinese traditional landscape, it's called "take advantage of a scene to express one's emotion". For example, the scene of "Moon over the Peaceful Lake in Autumn (ping hu qiu yue)" is used to express inner peace, and the scene of "Listen to the rain dropping on the leftover lotus (yu da can he)" is used to express the sentiment of sorrow. The atmosphere formed with the help of landscape can make the elderly residents resonate and enrich their inner world. With VR, these scenes can be reproduced more vividly without limitation from time and space (Table 3 ). 
Table 3. Design approaches for spiritual and cultural value based on the creation of landscape artistic conception

\begin{tabular}{|c|c|}
\hline Design approaches & Aims \\
\hline $\begin{array}{l}\text { Creating the natural landscape pattern } \\
\text { surrounded by mountains and rivers. }\end{array}$ & $\begin{array}{l}\text { To create a quiet and natural scene calms the mood, } \\
\text { and enhances the sense of environmental integration. }\end{array}$ \\
\hline $\begin{array}{l}\text { The application of Chinese traditional garden } \\
\text { landscaping techniques. }\end{array}$ & $\begin{array}{l}\text { To provide a variety of sightseeing experience and } \\
\text { maintains freshness. }\end{array}$ \\
\hline Creating traditional festival landscapes. & To lead to old people's recollection and identity. \\
\hline Creating Traditional cultural landscapes & To arouse a sense of cultural belonging. \\
\hline Creating country scenes & To arouse the memory and resonance. \\
\hline Using Traditional symbolic plants & To expresses the value recognition of the elderly \\
\hline
\end{tabular}

\section{CONCLUSION}

Smart Pension aims to cope with e severe problems caused by population aging with the help of information technology. It helps the elderly not only to improve their quality of life but also to help them demonstrate their value and dignity. Many scholars proposed that accommodating spiritual needs is of great significance to the seniors with Smart Pension in China. As an important expression of Smart Pension, the landscaping in retirement community is an indispensable part of the realization of Smart Pension. What are the types of the spiritual needs of elderly residents in the retirement community landscape and what kinds of expressions will be used to express them are the problems this paper tries to solve. Based on Customer Experience Theory, this paper takes the landscape design in retirement community in China as the research field, and concludes that the spiritual needs of the elderly residents in the retirement community landscape mainly include "emotional care" and "cultural care" in the context of Smart Pension, with "intelligent facilities and landscape" and "Festival and cultural landscape" as their representative expressions. We can promote intellectualization of facilities with the help of internet, cloud computing, Human-Computer Interaction, etc., to advance the practice of cultural and spiritual needs of the elderly, and to stimulate supports for social emotion, especially inter-generational relationship, cultural belonging, and self-realization, focusing both on material and mental development, and comprehensively enhancing user experience of retirement community.

Due to the relative lack of research on Smart Pension in retirement community in China, the research scope of this paper is small and the research depth is not enough. But as the initial exploration in this field, it has reference value. In the future, we will carry out the research on subdivision Association and application of intelligent technology according to demographic variables.

\section{ACKNOWLEDGMENTS}

This work is partially supported by Jiangsu Province Cultural research 2019 major projects, "Research on the innovation and development of cultural tourism industry with regional characteristics in Jiangsu Province in the context of Rural Revitalization"(Project No.19YB09).and Nanjing Institute of Technology research 2019 major projects, "Picturesque village construction on the construction of beautiful countryside in Jiangsu province"(Project CKJB201806) .

\section{REFERENCES}

[1] Xia Miaomiao. Current situation and hot spot analysis of smart Pension Based on CiteSpace bibliometric software $[\mathrm{J}]$. Modern Business Trade Industry, 2020, 41(32):85-87. DOI:10.19311/j.cnki.1672-3198.2020.32.037

[2] Zuo, M.Y. (2014). The connotation, mode and opportunity of smart pension. China Public Security, (10), 48-50. DOI: CNKI: SUN: GGAZ.0.2014-10-004

[3] Wang, Y, M. (2014). Study on Status and Problems of Information and Communication Technology (ICT) Intervening in Elderly Life in China. Scientific Research on Aging, 2(10), 42-51. DOI:10.3969/j.issn.2095-5898.2014.10.006

[4] Wang, Y, M. (2014). Network Society and Aging: Opportunities and Challenges. Academic Exchange, (8), 202-206.

DOI:10.3969/j.issn.1000-8284.2014.08.041

[5] He, C. Q., Tan, X. L. (2020). Review on the Interface Design of Digital Reading for the Elderly against the Background of Smart Pension. Packaging Engineering, (8), 202-206. DOI:10.19554/j.cnki.1001-3563.2020.20.010

[6] Bai, M., Zhu, Q. H. (2016). Analysis on the Current Situation of Smart Pension and Its 
Development Countermeasures.

Management Science, (9), 63-65. DOIi:10.3969/j.issn.1007-368X.2016.09.021

[7] Zhang, Q., Lee, H. (2019). From "how to be possible" to "how to be feasible" -- Research Progress and Enlightenment of smart pension abroad. Study and Practice, (2), 109-118. DOI:10.19624/j.cnki.cn42-1005/c.2019.02.013

[8] Bu, F. Q., Lee, M. Y. (2019). Investigation and design countermeasures of the elderly community landscape demand for the elderly. Journal of Shandong Forestry Science and Technology, (2), 54-57. DOI: CNKI: SUN: TREE.0.201902-014

[9] Hollebeek, L.D. (2011). Exploring Customer Brand Engagement: Definition \& Themes. Journal of Strategic Marketing,(Lead article), (7), 555. DOI:10.1080/0965254X.2011.599493

[10] Linda D. Hollebeek, L.D. (2014). Consumer Brand Engagement in Social Media: Conceptualization,
Scale Development and Validation. Journal of Interactive Marketing, (28) 149-165, DOI: 10.1016/j.intmar.2013.12.002

[11] Chen, Y. G., Liu, W. (2017). Factor Analysis of Plant Landscape Construction Based on the Perspective of the Elderly. Chinese Landscape Architecture, (08):115-118. DOI:10.19775/j.cla.2019.08.0115

[12] He, X., Zhu, L. S. (2018). Application of Colour in Outdoor Facilities for Senior Citizen. Design, (15), 12-13.

[13] Zuo, M. Y., Lei, D. Y.\&Shang, J. (2020). Demand and user analysis of smart pension service. Information China, (05), 38-41.

[14] Zhu, Z.R. (2018). Development prospects and development ideas of pension real estate. China Housing Facilities, (04), 87-89. DOI: 1CNKI: SUN: ZZSS.0.2018-04-038 